\title{
Automation in NS Monitoring and Alarm System
}

\author{
P. Maithili ${ }^{1}$, J. Kanakaraj ${ }^{2}$ \\ \{maithili.p.eee@kct.ac.in $\left.{ }^{1}, j k r @ e e e . p s g t e c h . a c . i n^{2}\right\}$
}

Assistant Professor, Dept. of EEE, Kumaraguru College of Technology ${ }^{1}$, Associate Professor, Dept. of EEE, PSG College of Technology, Coimbatore, Tamil Nādu ${ }^{2}$

\begin{abstract}
The person healthiness is enhanced from deficiency of liquids by providing NS (Normal Saline) to their health. Every time a NS is provided to the patients, the patient comprises to remain continuously supervised by nurse or a care- taker. In clinics there is a necessity of a somebody always to check the NS bottles every time the affected role is get admitted. There is an issue, when no one is present and monitoring the NS bottle level during dripping. It may cause serious issues to health by reversing the vein blood flow. In this paper NS level is monitored automatically and an alarm system is implemented to check the drip level availability limit. This proposed system permits a unconventional innovative method of modifiable NS rate via managing by retaining Internet of Things platform. In this proposed system, the load cell is used to check the saline rate. The new APP is developed to showcase our input. Arduino UNO is categorized such as microcontroller board with built on the ATMEGA328P.
\end{abstract}

Keywords: NS (Normal Saline), Fluid level, Level Sensors, IoT.

\section{Introduction}

In modern period, nearly all of the system remains developing, and the innovative globe is making progress furthermore. An Advanced system technology is Internet of Things (IoT). A smart system is designed and developed by interlinking the sensors and other devices using IoT technique. This IoT is applied in automation of home, physical condition supervision systems. This type of monitoring systems is used to monitor the human essential parameters like HBs, Sugar level, Hemoglobin and pressure level. The "smart beds" are available in most of the health care unit to have a follow up on the parameters like temperature, pressure and heartbeat rate, sleep rate. Similar way the saline level of the patient is monitored by the proposed IoT based smart system. The saline is nourished to the peoples those who have deficiency in liquid level. Nonstop observing of saline quantity is pretty significant work, somewhat error ended while of nursing, in forthcoming patient might undergone some health problems or complications. This system might be dangerous to the people health else it could harm the nerve system of patients. Thus, supervising of saline quantity is the accountability of one clinic workers such as Doctors, nurses, care- taker. In utmost cases clinic or hospital nurse fails to check the saline quantity repetitively. Hence it leads the dangerous to human life. Therefore, to sidestep this condition the proposed system is used for monitoring level of saline constantly and automatically. To check the weight of NS bottle, it has a stand which carries a load-cell. An alarm is produced with alarm message in LCD display, once the saline level in the NS bottle is over. It send the alert message to the corresponding care taker of patient. The 
benefit of this scheme is that nurse can proficiently work and give appropriate time to every single patient.

\section{Existing System}

An existing procedure followed in hospitals requires nurses to check the NS bottle level frequently and give proper care to each patient. Normally, roller wheel clamp appear at every single NS container is utilized for regulating the flow level of the NS bottle, with presence. The whitecap clamp is trolled in opposite to the clockwise track, to bandages the circulatory pipe further rigidly which make it to thinner follow-on in to sluggish down the movement rate of NS liquid. The system releases the saline water with fast flow rate when the roller wheel clamp switched in clockwise direction [3]. Presently, this type of system is not available in hospitals. It lessens the need of nurses to monitor the patient's saline level constantly. This proposed system diminish the necessity for the nannies to go to patient role cot every single period to checked NS level position of individual patient. It's a time to develop an advanced, low cost and efficient glucose water bottle monitoring system with IoT.

\section{Proposed System}

The flow rate and glucose bottle level is measured by a new method in this proposed system. In this system the load cell used in the direction of detecting the mass of NS bottle. The definite mass of the NS bottle is weighing, and this value noted as $100 \%$ filled state. The real weight of the NS bottle is decreased to the level to $10 \%, 25 \%$ and $50 \%$ is displayed in LCD. This has been connected to the website through Arduino and IoT. The flow will be automatically cut off and the buzzer will alert when the level reaches $10 \%$. The care- taker can control the flow rate with web portal. The flow of blood from patient into the saline bottle is prevented by this proposed system.

The block diagram of proposed system connection is shown in fig.1.

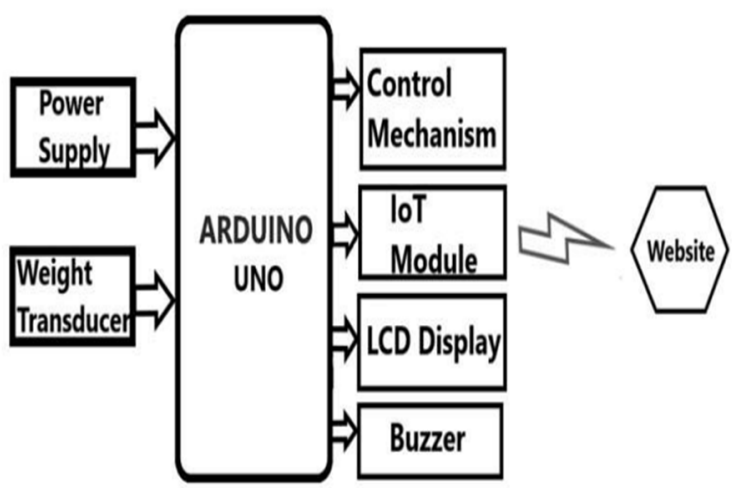

Fig.1 Block Diagram of Proposed System

This proposed system will give the analog value of the weight of NS bottle through the assistance of Load Cell. The analog to digital conversion is done by load cell amplifier 
(HX711) and the digital value is given to the microcontroller the level can be seen from website and also from LCD display. The alarm sound is giving awareness to the nurse or caretaker about the low level of the saline bottle i.e. 10\%. This flow can be stooped from website automatically.

The points mentioned below are the proposed system objectives:

1. The dis-advantages of existing system is to be conquered.

2. The system has better précised output than the existing system.

3.In manual saline control system, If the nurse is not available it hurts the patient once the saline bottle gets empty.

4. To make the NS level supervising automatic and to Notify the caretaker instinctively for patient wellbeing.

5. This proposed system will cut off the saline flow when the bottle becomes empty.

\section{Methodology}

The proposed system flow chart is shown in fig.2. Details of the saline bottle level and temperature of the human body is sensed and monitored. These details fed into the IoT and its delivered into the application through Bluetooth. Then all the details related to this system is monitored in real time using the app used in mobile. The sensors is used to check the liquid range of the glucose bottle by sensing moisture content of it. The range and flow rate of the fluid level is sensed and controlled automatically. If the nurse is far away from patients' bed, he/she will analyze the fluid level data using mobile app. It gives an alert message to the nurses or care- takers immediately. Once the fluid level goes to critical value, the system will notify the status of the glucose liquid level and patient condition to the doctors.

The reverse flow of blood from vein to saline bottle is prevented by this proposed system. This system will have the cut off technique to stop and control the fluid level of saline bottle. There will be weight transducer, it will check the weight of the glucose bottle. Once the liquid is entirely/completely fed into the patient this system will activate the control mechanism that stops the liquid flow from saline bottle. It gives better benefit to the patient. 


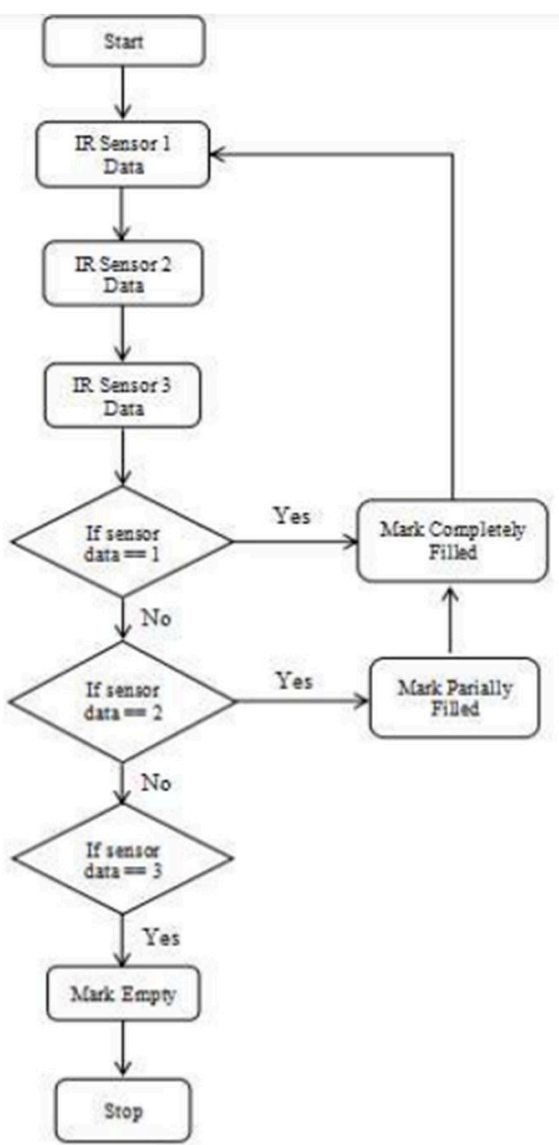

Fig.2 Flow chart of Proposed System

\section{Components used in this System}

Four elements utilized in this scheme are:

1. To monitor status of fluid level and status of patient, Sensors fixed

2. Module for conditioning of sensor signals

3. Supervising Module for Patient

4. Handy mobile for doctors/nurses

1. Sensors fixed to the Patient and load cell on Saline bottle:

Inserting the beat device, Instrument used to measure temperature, Blood Pressure measuring device(sensor), Liquid range sensor through the Arduino board.

a) Temperature Sensor: Patient physique temperature is measured by LM35 sensor. It is fixed to the patient's body to measure the temperature level. The sensor is standardized with Celsius. An external standardization is not required for this proposed system. This system has small self-heating ability. 
b) Load Cell: It is a device which produce an electric output signal by sensing the force or tension. There will be a change in resistance that leads the modification in the output voltage of the sensor. If the force is applied into the sensor/transducer the resistance is changes as per the input.

2. Section for conditioning the signals:

a) Arduino Uno:

Arduino Uno is categorized such as microcontroller board built on the ATMEGA328P. This One incorporate 14 input/output digital pins. Among these 14pins, PWM output utilizes 6 and analog input uses 6 . It includes USB cable, reset switch and power card. This system is get connect with PC through USB cable and it will assist the microcontroller. This will be supplied by the converted AC-DC adapter and battery.

b) Bluetooth Module: There are two operating method in Hc05.In first mode the data can be transmitted and received from external Bluetooth devices. In second mode, there will be the possibility to change the default settings.

3. Supervising Module for Patients

The data from the users and environment is collected consistently by using IOT devices. This One shared to the application through Bluetooth. The real time data's like liquid level of saline bottle, Blood Pressure, heart-beat rate, temperature of the patient is monitored by this system. These data are compared with the preset values. It will give alert message to the nurses if the fluid level goes below the preset value.

4. Mobile of Doctors/Nurses

The Proposed system continuously supervising the saline level and patient body condition and its send to the nurses/ doctor's mobile in detail. Instantaneous patient monitoring is achieved with this system.

\section{Hardware Implementation}

The hardware diagram of the proposed system is shown in Fig.3.

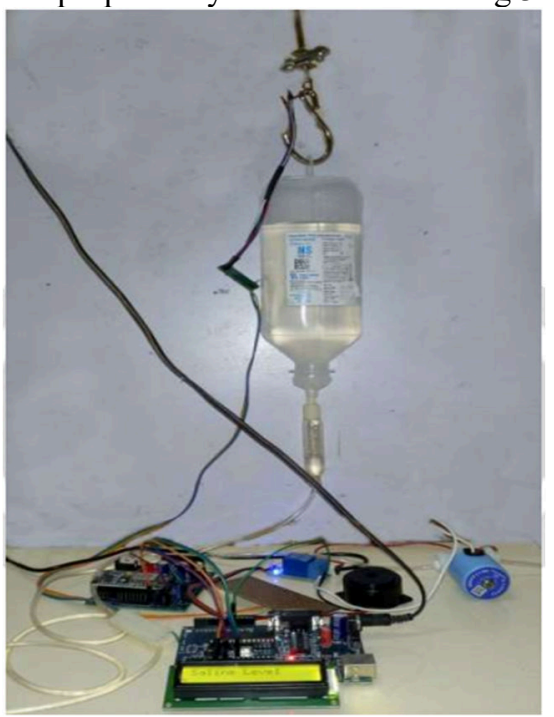

Fig.3 Hardware of Proposed System 
The hardware was developed and verified the results obtained. The hardware was demonstrated for no load and load condition also. The No load output is shown in Fig.4.

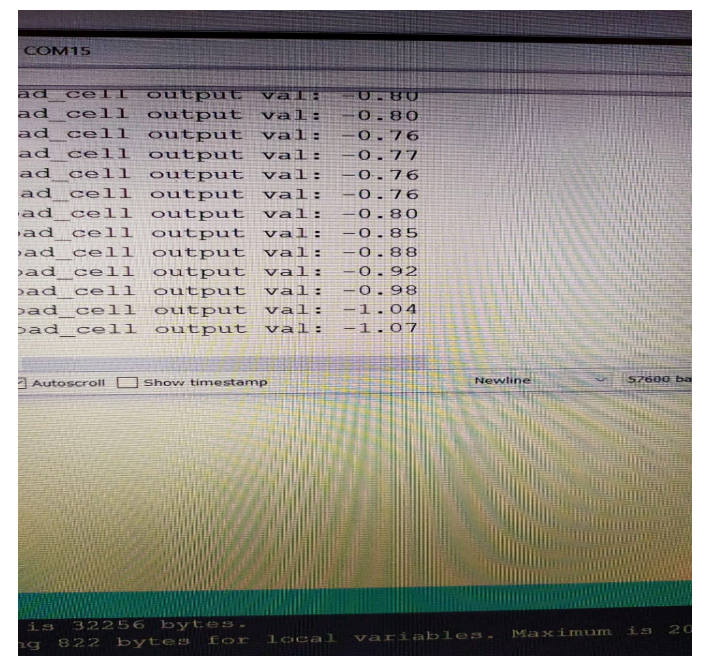

Fig.4 Hardware output of Proposed System at No load condition

This System shows the value in different levels.

\section{APP Implementation}

The APP development and integrate it into the proposed system is explained. The icon of the NS monitoring and alarm system is shown in Fig.5.

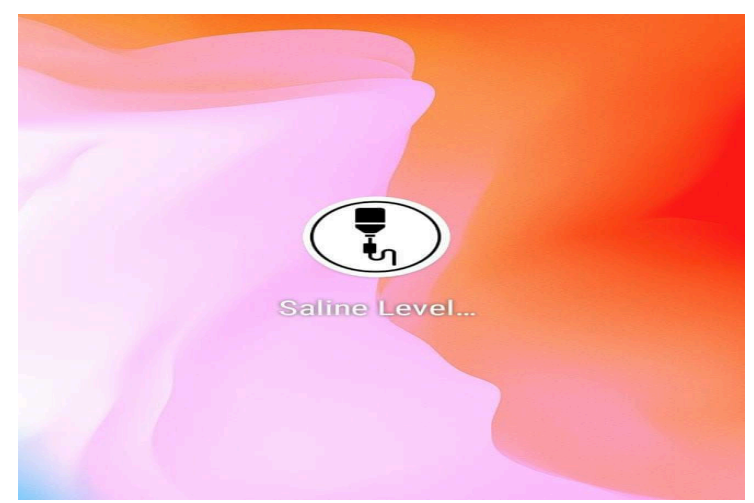

Fig.5 APP ICON

App indicating the rooms of a hospital and level of saline in each room in percentage. The developed App shows that the level of NS in each room with different colours. In this system, initially four rooms are included in the App for demonstration. The NS level of each room is monitored uninterruptedly, and the results were shown. Then how can change the patient's 
name is also explained further. It is possible to add/remove the rooms or patients name in the App without any tedious work.

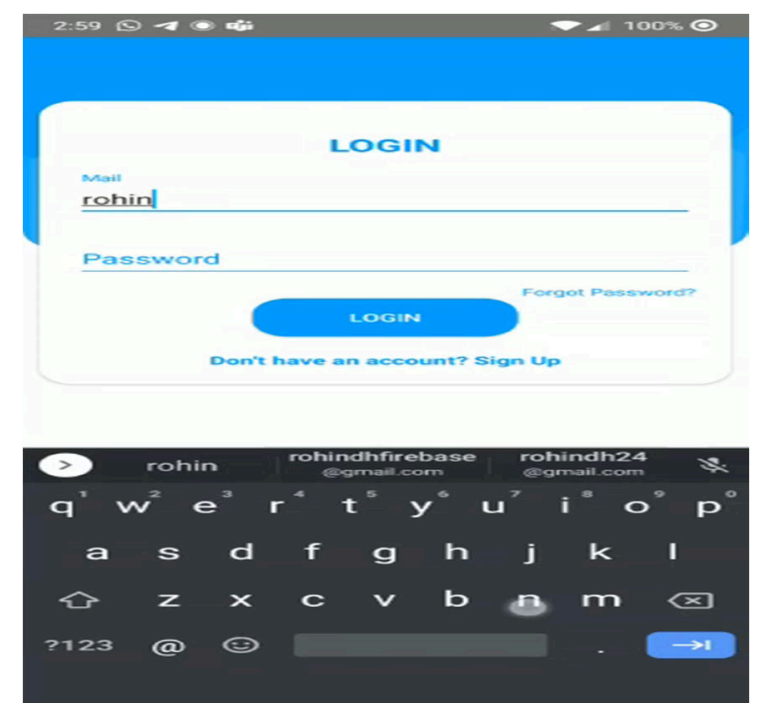

Fig.6 App Login Page

The Log in pages of the developed App is shown in Fig.6. It is very simple as like our regular mail. Initially we need to give the username along with password to get into the home page of the App. Each care takers and doctors can create their own account in this App. The App implementation is shown in Fig.7.

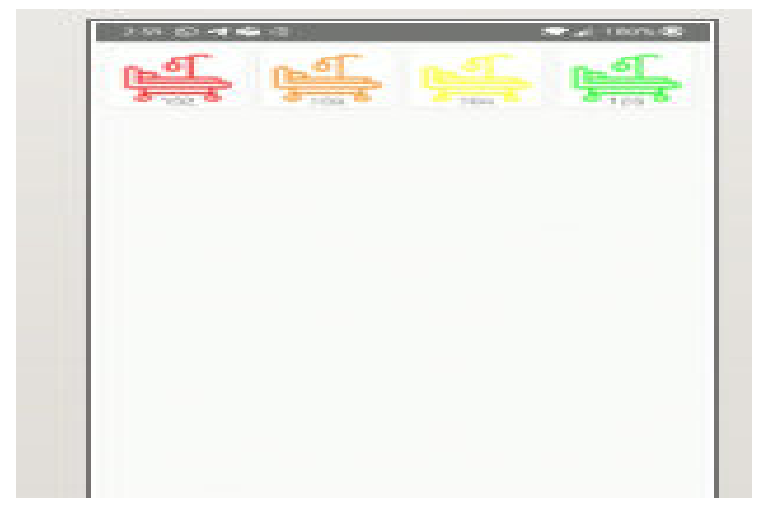

Fig.7 App Implementation

Initially Room No:102,103,104 and 105 is included in the system. The patient Names of each room is mentioned in the system. The NS level monitoring output of each patients is shown in Fig.8 and 9. 


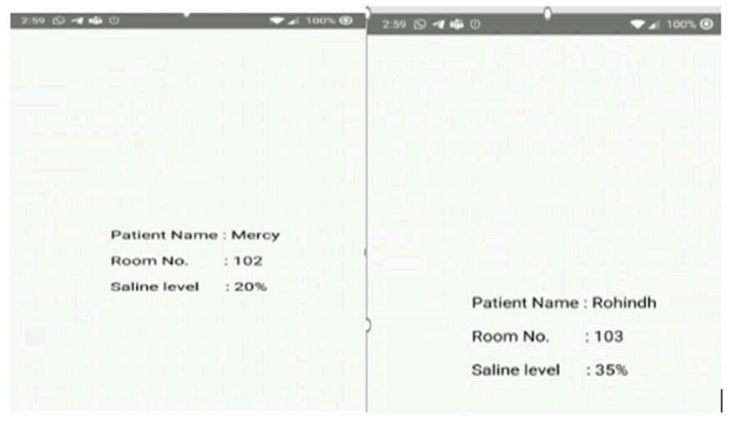

Fig.8 NS Monitoring Output of the Room No:102 and 103

The Saline level other two patients are observed and the output is shown below.

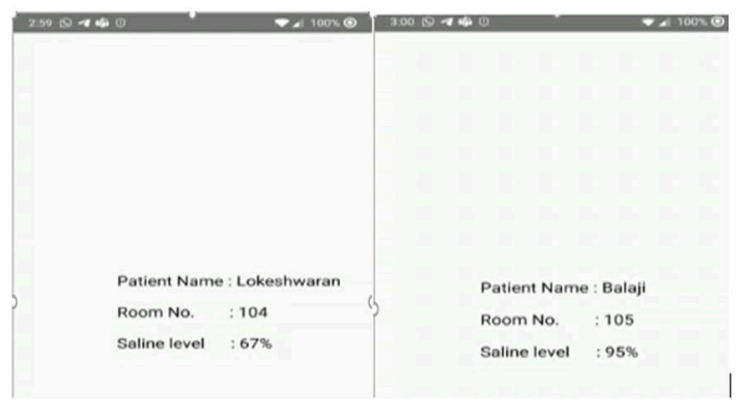

Fig.8 NS Monitoring Output of the Room No:104 and 105

In Room No.102 the saline level monitored and shown the value as $20 \%$ and in Room No:103 the output is shown as 35\% and other two patient values are around $67 \%$ and $95 \%$. These values are reflected into caretakers display once they have looged into the account. Hence the nurses or doctors can supervise the patient NS level frequently.

Now, the old patient was discharged, and new patient is allotted at Room No:105. This changes also updated into this system automatically. The new rooms addition and deletion done easily in this App and it will reflected into display. The new patient inclusion is shown in Fig.9. 


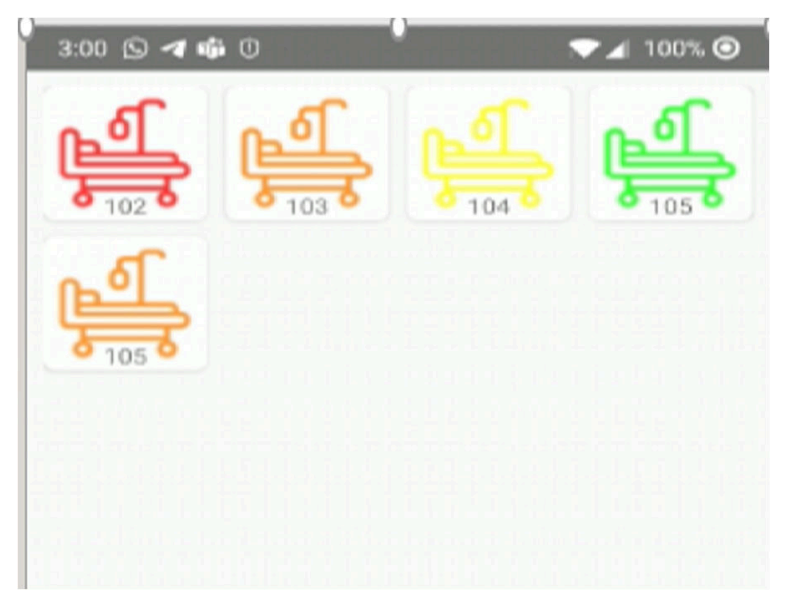

Fig.9 New Patient details added into the existing Room:105

The non-stop saline level observing output of this Room No:105 is shown in the display. The initial value of NS level of this patient is shown as $47 \%$ in Fig. 10 . Then the level of liquid is reduced to $23 \%$ and finally it reaches $10 \%$.

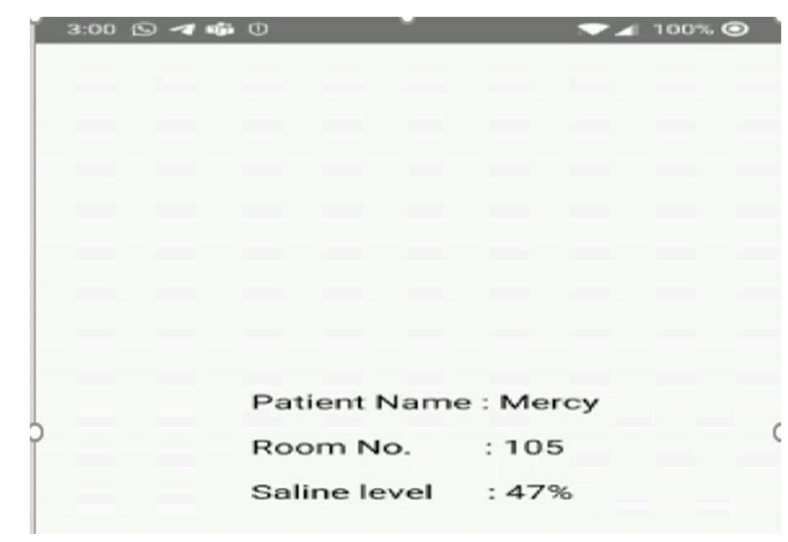

Fig.10 First value of NS Monitoring Output at Room No:105 
Fig.11 Second NS Monitoring Output of the Room No:105

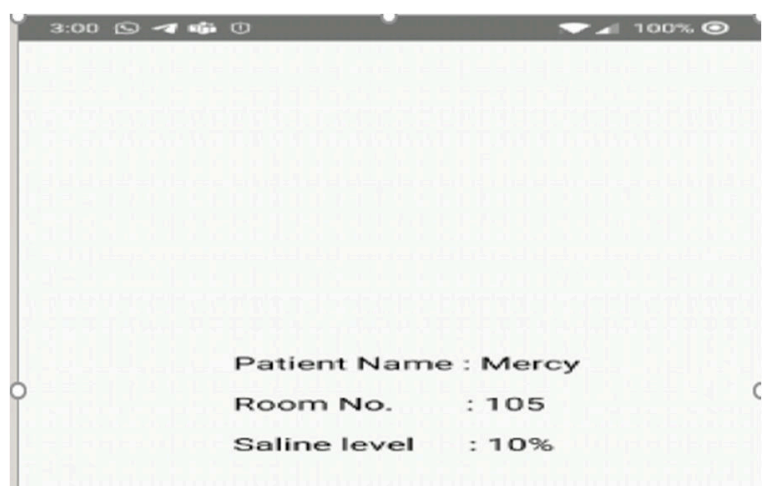

Fig.12 First NS Monitoring Output of the Room No:105

IoT based NS level monitoring system reduced the labor-intensive determination on the share of the nurses. This system is beneficial for the caretakers by the side of nighttime. This programmed NS level observing system affords an extra flexibility to doctors, thereby the care on patients is improved. The system requires low cost, reliable and accessible to care- takers or nurses. It can be reused for the next NS bottle. The nurses does not needed to visit the patient's place in order to confirm the amount of NS present in the glucose bottle and flow rate.

\section{Conclusion}

An automatic Glucose bottle level monitoring system uses IoT to reduce the nurse and patients struggle. This new system requires no manpower because it is automatically cut off the saline flow rate. The patient lifetime is protected by this system. It will be useful for the nurses to supervise the patient's saline level without going into the patient's bed each time. If the saline level goes below or reaches the critical level, the system will send an alarm signal to nurses, doctors or caretakers. Strain of nurses or doctors is minimized from continuous monitoring of patients by this new cost-effective method. This automatic NS level checking 
scheme affords additional flexibility to specialists, in that way the patient's safety is improved. Henceforth it reduces the working and monitoring time of nurse and doctors. The system requires low cost, reliable and accessible to care- takers or nurses. Use it again subsequently to the new saline container. The proposed scheme assists nurses to screen the NS movement from a remoteness. This system is more beneficial during night-time. The nurses do not needed to visit the patient's place to confirm the amount of NS present in the glucose bottle and flow rate.

\section{References}

[1] Rashid, H., Shekha, S., Reza, S. T., Ahmed, I. U., Newaz, Q., \& Rasheduzzaman, M. (2017, February). A low cost automated fluid control device using smart phone for medical application. In 2017 International Conference on Electrical, Computer and Communication Engineering (ECCE) (pp. 809-814). IEEE.

[2] Yuan Jie Fan, Yue Hong Yin, Member, IEEE, Li Da Xu, Senior Member, IEEE, Yan Zeng, and Fan Wu," IoT-Based Smart Rehabilitation System", IEEE TRANSACTIONS ON INDUSTRIAL INFORMATICS, VOL. 10, NO. 2, MAY 2014,Page No.1

[3] Digarse, P. W., \& Patil, S. L. (2017, June). Arduino UNO and GSM based wireless health monitoring system for patients. In 2017 International Conference on Intelligent Computing and Control Systems (ICICCS) (pp. 583-588). IEEE.

[4] Lei Yu, Yang Lu, XiaoJuan Zhu,\| Smart Hospital based on Internet of Thingsll, JOURNAL OF NETWORKS, VOL.7, NO. 10, OCTOBER 2012, Page No.1-8.

[5] Ghosh Debjani, Agrawal Ankit , Prakash Navin , Goyal Pushkal (2018)"Smart Saline Level Monitoring System Using ESP32 And MQTT-S"2018 IEEE 20th International Conference on eHealth Networking, Applications and Services (Healthcom).

[6] Majumder, S., Rahman, M. A., Islam, M. S., \& Ghosh, D. (2018, September). Design and Implementation of a Wireless Health Monitoring System for Remotely Located Patients. In 2018 4th International Conference on Electrical Engineering and Information \& Communication Technology (iCEEiCT) (pp. 86-91). IEEE.

[7] Chowdary, K. C., Krishna, K. L., Prasad, K. L., \& Thejesh, K. (2018, August). An efficient wireless health monitoring system. In 2018 2nd International Conference on I-SMAC (IoT in Social, Mobile, Analytics and Cloud)(I-SMAC) I-SMAC (IoT in Social, Mobile, Analytics and Cloud)(I-SMAC), 2018 2nd International Conference on (pp. 373-377). IEEE.

[8] D. S. Vijayan, A. Leema Rose, S. Arvindan, J. Revathy, C. Amuthadevi, "Automation systems in smart buildings: a review", Journal of Ambient Intelligence and Humanized Computing https://doi.org/10.1007/s12652-020-02666-9

[9] Kiruthika, C., S. Lavanya Prabha, and M. Neelamegam. "Different aspects of polyester polymer concrete for sustainable construction." Materials Today: Proceedings 43 (2021): 1622-1625. 